\title{
Telehealth at UC Davis-A 20-Year Experience
}

Thomas S. Nesbitt, MD, MPH, ${ }_{1}^{1,2}$ Madan Dharmar, MBBS, PhD, ${ }_{1}^{1,3}$ Jana Katz-Bell, $M P H_{1}{ }^{1}$ Gunnar Hartvigsen, $P h D_{1}^{4,5}$ and James P. Marcin, MD, MPH ${ }^{1,3}$

${ }^{1}$ Center for Health and Technology and ${ }^{2}$ Department of Family Practice and Community Medicine, University of California Davis Health System, Sacramento, California.

${ }^{3}$ Department of Pediatrics, University of California Davis Children's Hospital, Sacramento, California.

${ }^{4}$ Norwegian Centre for Integrated Care and Telemedicine, University Hospital of North Norway, Tromsø, Norway.

${ }^{5}$ Department of Computer Science, University of Tromsø, Tromsø, Norway.

\begin{abstract}
Telehealth at the University of California Health System began as a telefetal monitoring connection with a rural hospital in 1992 and evolved to become the Center for Health and Technology (CHT) in 2000. The Center supports the vision of the University of California Davis (UC Davis) Health System-a healthier world through bold innovation. The CHT focuses on the four pillars of the academic health center: clinical services, research and scholarly work, education, and public service. Since 1996, the Center has provided more than 33,000 telemedicine consultation (excluding teleradiology, telepathology, and phone consultations) in over 30 clinical specialties and at more than 90 locations across California. Research and continuous evaluation have played an integral role in shaping the telehealth program, as well as strategic collaborations and partnerships. In an effort to expand the field of telehealth the CHT provides telehealth training for health professionals, technical specialists, and administrators. Furthermore, it also plays an integral role in workforce development through the education of the next generation of community primary care physicians through Rural Programs In Medical Education (Rural PRIME) and continuing educational programs for working health professionals through videoconferencing and Web-based modalities. The Center is supported through a variety of funding sources, and its sustainability comes from a mix of fee-forservice payment, contracts, grants, gifts, and institutional funding. Together with key partners, UC Davis has educated and informed initiatives resulting in legislation and policies that advance telehealth. Looking toward the future, UC Davis is focused on technology-enabled healthcare and supporting synergy among electronic health records, health information exchange, mobile health, informatics, and telehealth.
\end{abstract}

Key words: telemedicine, telehealth, research, distance education, home health

\section{Introduction}

he University of California Davis (UC Davis) Health System (UCDHS) is a 619-bed, fully integrated academic health system whose mission is to improve lives and transform healthcare through innovation. Being the region's only academic health center, it serves around 6 million residents in 33 counties spread across a 65,000 square mile area that stretches north to the Oregon border and east to the Nevada border. UCDHS is the only Level 1 adult and pediatric trauma center in Northern California and has over 1 million outpatient and emergency room visits with around 31,000 patient admissions each year. The health system strives to improve the health of the residents in its community and the rest of California through innovation, discovery, and knowledge sharing.

Although the telemedicine program began in the mid-1990s, the Center for Health and Technology (CHT) was formally established in 2000 (Fig. 1). The vision of the CHT is to create an environment that brings together innovative technologies with the expertise of physicians, nurses, educators, information technology and communications specialists, engineers, and researchers. Collectively, these teams work together to improve access and quality of patient care. The CHT has three goals: (1) to support the Health System's clinical outreach mission using telehealth and distance education: (2) to provide an environment that fosters collaborations among stakeholders; and (3) to serve as a telehealth resource in areas of information and telecommunications technology for the Health System and its affiliates, other health systems and providers, government agencies, and industry partners. Today, the Center serves as a telehealth hub for medically underserved communities throughout California.

The clinical telehealth program has generated approximately 33,000 inpatient and outpatient encounters (excluding teleradiology, telepathology, and phone consultations) since 1996. The majority of the encounters utilize two-way video and audio, with the exception of teledermatology, telecardiology, and telepsychiatry (which offer both real-time and asynchronous consultations) and retinopathy screening (asynchronous) (Fig. 2). UC Davis provides services to clinical partners within California and out of state and to a limited number of international clients. However, 90\% of the telehealth services are provided to patients residing in Northern California. Approximately 50\% of the consultations were for patients who live more than 100 miles from Sacramento. About one-quarter of the patients reside in rural regions, another quarter reside in urban areas, and about half of the historical encounters were to correctional patients. These proportions are shifting with a decreasing number of correctional patients. The most utilized real-time specialties are 


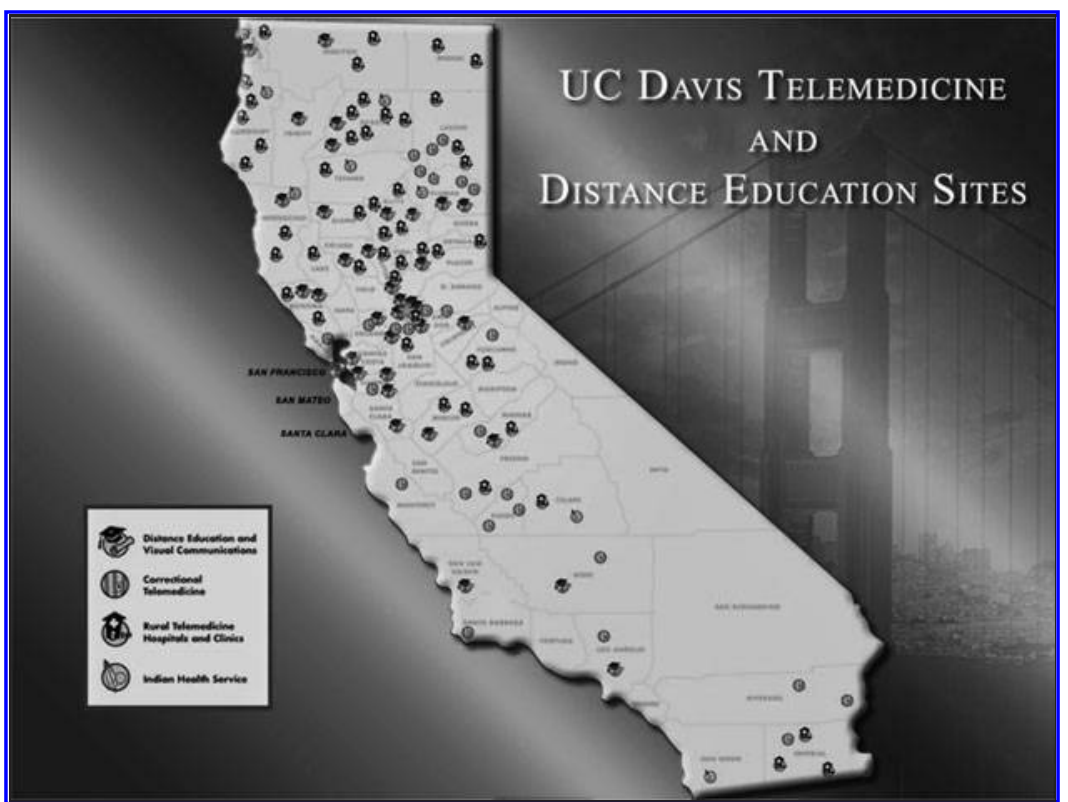

Fig. 1. University of California Davis Health System, Center for Health and Technology. the Office for Rural Health Policy and another one from U.S. Department of Agriculture Rural Utilities Service Distance Learning \& Telemedicine Program in 1997, which enabled it to expand its telehealth services to surrounding rural hospitals.

UC Davis established a formal telehealth educational curriculum in 1999 with funding from The California Endowment (a California-based health foundation). This program is designed to provide education for healthcare providers, technical specialists, and administrators on how to launch and/or expand successful telehealth programs. In 2000, the UC Davis executive leadership approved the creation of the CHT as a formal center with a broader mission around clinical service, research and development, industry relations, and education. The Center's role further expanded in 2004 as a resource for the State of California when it received a biodefense training and preparedness contract for the State focused on the use of telehealth and related technologies. Since the mid-2000s, UC Davis has expanded clinical offerings and revenue streams, developed a broad telehealth research portfolio, and focused on strategic partnerships to advance the field. dermatology, psychiatry, soft-tissue orthopedics, endocrinology, and hepatology. The pediatric critical care telehealth program, although small in volume, is a visible component of the CHT.

The UC Davis program started in 1992 under the leadership of Dr. Thomas Nesbitt with a telefetal monitoring program between the campus and Colusa Community Hospital, 70 miles away from Sacramento. This simple, analog connection supported a partnership between the family medicine physicians in Colusa and the perinatologists at UC Davis and re-established obstetrical services that had been unavailable in the county for over 5 years. In 1996, UC Davis implemented video-based, telehealth service, connecting three specialties with three remote sites. UC Davis received its first grant from

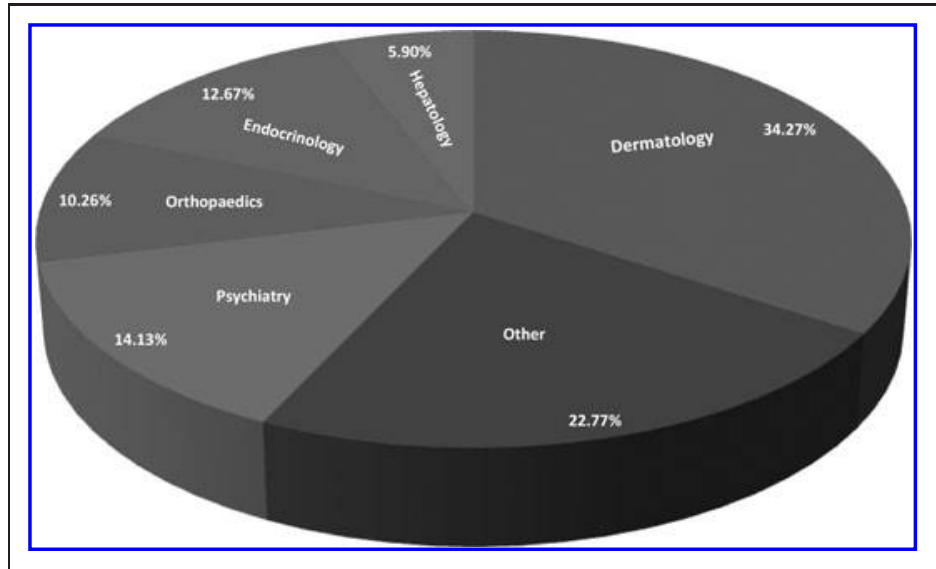

Fig. 2. Clinical specialties: synchronous outpatient telemedicine consultations.

\section{Role of Telehealth in the UCDHS}

Telemedicine at the UCDHS primarily focuses on the four mission areas of clinical service, research and scholarly work, education, and public service. The program has diversified in the past two decades to include over 30 clinical specialties, pediatric emergency outreach, correctional telemedicine, Indian Health and frontier telehealth, distance education and media production, virtual tumor board, teleaudiology, sexual abuse evidentiary examination quality assurance, telepharmacy, tele-interpreting, services for military families (Family-Link), and disaster preparedness. UC Davis has been part of several ad hoc international telemedicine consultations and has piloted the use of telemedicine for immigrant farm workers. In addition to providing specialized care to rural communities, UC Davis also provides lifelong learning opportunities to rural physicians, nurses, and other healthcare professionals through its distancelearning and online classes.

\section{CLINICAL SERVICES}

One of the main goals of the clinical program is to increase access to quality healthcare, particularly in rural and/or underserved communities. The program serves a diverse patient population throughout the State of California, including both adults and children. The clinical program is fairly distributed across its network, providing services mainly in rural and/or safety net clinics, some correctional facilities (federal and state prisons, jails, and juvenile detention centers), Indian Health Service sites, and critical access and/or public hospitals. The majority of the clinical visits are scheduled outpatient visits with the specialist connecting from the telemedicine clinic to a rural clinic or correctional facility. 


\section{TELEHEALTH AT UC DAVIS-A 20-YEAR EXPERIENCE}

In the inpatient area, pediatric emergency outreach provides a hospital-based acute care telemedicine service in emergency departments and adult intensive care units in rural hospitals. The acute care telemedicine service is provided by a pediatric emergency or critical care medicine physician to rural hospitals, to provide timely specialist care to critically ill or injured children over telemedicine. ${ }^{1,2}$ Several benefits are realized through this program, including a higher level of care in the community, a reduction in transportation cost, and an increased availability of Pediatric Intensive Care Unit beds in the UC Davis Children's Hospital in Sacramento for critically ill patients needing a high level of care. In addition, pediatric subspecialists offer their expertise to the physicians treating children admitted in hospital wards at local community hospitals in Sacramento. Since its inception in 2000, pediatric specialists from the UC Davis Children's Hospital have provided over 300 pediatric emergency and critical care telemedicine consultations to 14 rural and underserved emergency departments, 2 community hospitals' pediatric inpatient wards, and another community hospital's adult intensive care unit. Similarly, the adult infectious disease group serves understaffed hospitals in California treating patients with human immunodeficiency virus infection, viral hepatitis, fungal infections, complicated urinary tract infections, chronic osteomyelitis, and immune deficiency.

\section{RESEARCH}

The CHT along with the Clinical and Translational Science Center, School of Medicine, and School of Nursing have established a robust environment to promote innovative research in medicine. This Center has served as a resource for innovative research projects focused on improving the quality of healthcare.

\section{EDUCATION}

The Telemedicine Education Program (TEP) was established in 1999. Its primary goal was to train healthcare professionals, technical support personnel, and administrators to develop and sustain a successful telehealth program in the community. With the formation of the CHT, the TEP became an integral part of the Center. Its facility offers a customized 30-seat classroom, with fully equipped telemedicine training laboratories including mock examination rooms and consultation suites. The TEP embraces a multidisciplinary, adultlearning approach to its curriculum, incorporating discussions, lectures, and hands-on training. More than 1,750 people from over 800 organizations have attended the training courses. Courses are offered four times a year, one of which is at the American Telemedicine Association's annual meeting, while the others on campus.

Telehealth is also an integrated component of an educational track in the School of Medicine called Rural Programs In Medical Education (Rural PRIME)-one of five specialized curricula within the University of California medical school system. The program is designed to train physicians for rural practices and offers a range of educational content, including public health issues and the use of medical technologies such as telemedicine. It has training sites in rural areas of the Central Valley, foothill region, and northern reaches of the state. Students remain in contact with their School of Medicine instructors in Sacramento via videoconferencing connections to maintain the continuity of curriculum and learning from distant locations. Telemedicine consultations are integrated into their training program, resulting in expanded services to the Rural PRIME rural sites and also preparing Rural PRIME medical students in this technology as a support for their future practices.

The CHT includes distance education within its portfolio and is committed to deploying technology that supports lifelong learning to diverse health professionals. Content is delivered by many modalities, including streamed video, videoconferencing, and other online tools. Examples include weekly distribution of Grand Rounds, special sessions requested by partner clinicians (a "You Asked for It" series sponsored by Continuing Medical Education), and online education. Departments within the Health System utilize the CHT to support international linkages to Brazil and India. The CHT has more than 500 courses available through various technologies such as interactive videoconferencing, interactive webcasting, and on-demand video for convenient distance learning.

The National Cancer Institute-designated Cancer Center at UC Davis uses telehealth to offer a Virtual Tumor Board. This project is part of the UC Davis Cancer Care Network covering Northern and Central California. The Virtual Tumor Board enables the multidisciplinary teams involved in patient care to meet in real time using state-of-art telemedicine technology, share their medical knowledge, and agree on the best treatment plans for the patients. Patients receive care in their own communities and from their own physicians, who are in collaboration with an academic health system and colleagues around Northern California. Experts in hematology and oncology, surgical oncology, radiation oncology, pathology, diagnostic radiology, and other specialties work together to develop individualized treatment plans for each patient. At the same time, they conduct research on cancer risks, treatments, and prevention.

The Tele-Audiology Program is a new interdisciplinary collaboration among UC Davis CHT, the Departments of Pediatrics and Otolaryngology, the Children's Medical Services Branch at the California Department of Healthcare Services, and a medical center in Redding, CA (140 miles north of Sacramento). This program uses telehealth technologies to allow a UC Davis audiologist in Sacramento provide comprehensive audiological diagnostic services to infants in Redding. It aims to address the unacceptably high "lost-tofollow-up" rates for infants who have failed their newborn hearing screen, obviating long travel and wait times to access qualified audiologists. This can have a positive effect on the child's cognitive and developmental milestones.

Another application is a quality assurance program for sexual assault examinations. Physicians and nurse practitioners at the Child and Adolescent Abuse Resource and Evaluation Center at the UC Davis Children's Hospital provide 24-h child abuse evidentiary evaluation and consultation services via interactive videoconferencing with patients, physicians, and child abuse specialists in six rural hospital emergency departments in underserved regions of Northern California. The program also provides monthly child abuse 


\section{NESBITT ET AL.}

training to healthcare providers in these hospitals who might otherwise forgo such opportunities because of time and travel distances required to attend on-site training.

UCDHS piloted a telepharmacy program, connecting six rural hospitals in Northern California to pharmacists at UCDHS in Sacramento. Although these hospitals had on-site pharmacies, they did not have the resources or capacity to staff a pharmacist after regular business hours. The nurse from the remote hospital now connects with the UC Davis pharmacist via videoconferencing who reviews the medication orders and medication labels and verifies the medication and strength.

\section{PUBLIC SERVICE AND COMMUNITY ENGAGEMENT}

Tele-interpreting services are available to UC Davis patients in more than 16 languages and dialects. On average, the Health System provides over 100 tele-interpreting encounters each day. Trained medical interpreters convey the meaning of the source language using specialized terminology and idioms over videoconferencing.

Family-Link is a unique program supported by the CHT and UC Davis Children's Hospital staff that uses low-cost telehealth technologies to connect hospitalized pediatric patients and their families at the bedside for those who are not able to visit in the hospital, thereby diminishing the stress on the family that may result from long hospitalizations, particularly for children.

\section{Analytical and Empirical Information}

Research and continuous evaluation are integral elements of the CHT. Several faculty members focus on telehealth as their primary research area. The goal is to utilize telehealth technologies within clinical trials to measure outcomes, to ascertain qualification for clinical trials, and to triage patients. In 1996, the UC Davis started a telemedicine pilot program at three of its primary care network sites comprising both urban and suburban clinics. ${ }^{3}$ Investigators at UC Davis reviewed the first 1,000 consultations to assess the start-up experience by comparing the demand and satisfaction scores of rural and urban or suburban clinics. ${ }^{3}$ In 2002, CHT researchers reported improved quality from outpatient telemedicine specialty consultations to primary care clinicians. ${ }^{4}$ This study also found that telemedicine consultations resulted in changes in diagnosis and treatment regimens as well as improvement in clinical outcomes, ${ }^{4}$ demonstrating that telemedicine has the potential to increase the quality of healthcare in local communities. In 2004, Marcin et al. ${ }^{2}$ documented positive clinical outcomes of risk-adjusted quality as well as high satisfaction in inpatient pediatric critical care telemedicine consultations. In 2004, Marcin et al. ${ }^{2,5}$ also reported overall cost savings for regional healthcare and increased revenue for the rural hospital (adult intensive care unit) from this program.

In 2005, Nesbitt et al. ${ }^{6}$ found that telemedicine outreach into rural communities was associated with an increase in the local communities' perception of local healthcare quality. A recent study with the Center for Connected Health Policy entitled the Specialty Care Safety Net Initiative, which included all 5 University of California medical campuses and 40 community clinics, found that the overall costs of operating the telemedicine clinics were, on average, less than the traditional in-person clinics. This cost was higher in a few clinics, particularly those with very high costs of physician time. The cost of providing telemedicine services was generally higher than the reimbursement from the state's Medicaid program, Medi-Cal, because of its low reimbursement rate. However, if a Federal Qualified Health Center prospective payment model or a cost base reimbursement model was used, the cost of care delivery was well compensated by this model. The increased focus on telehealth research by UC Davis has played an important role in shaping our telehealth program as well as collaborations and partnerships.

\section{Sustainability Planning}

The CHT is supported through various revenue sources, including fee-for-service payments, contracts, grants, gifts, and institutional funding. The UCDHS invests in telehealth services as an approach to fulfill goals in research, clinical service, education, and public service. The CHT and its telemedicine program have received financial support from the Health System since the beginning of the program. This line item in the base budget supports human resources and infrastructure (clinic support staff, technical staff, media production, licenses and maintenance, and business functions). In 2005, the UCDHS Department of Finance evaluated the revenues to the Health System from communities following the establishment of telehealth partnerships. The data indicated that telehealth partnerships influenced the overall revenue to the Health System, and the service was valued by the clinical partners in the broader community. A similar finding was observed in the pediatric emergency and critical care program. These findings were reported to internal decision makers and executives as part of ongoing discussions regarding the strategic role of the CHT. The most common financial model for consultative clinical services is to bill third-party payers (fee-for-service) and to execute contracts for scheduling blocks with telehealth partners. Initially, the telemedicine program established agreements with departments to cover the cost of faculty time. The telemedicine program assumed the financial risk by billing the third-party payer or the contracted facility once the service has been rendered. This model was reflective of the novelty of telehealth in the mid/early 1990s. With the positive reimbursement policies in California, this model has now changed, with each individual department bearing the risk for recovering the cost of their provider's time from the third-party payer or contracted hospital/clinic. The CHT continues to support outreach efforts by providing administrative, billing, and technical assistance, as well as support through institutional funding.

Grant funding has been essential to achieve the vision of CHT. Since 1996 , we have received nearly $\$ 100$ million in extramural funding for various projects related to telehealth. Foundations (California HealthCare Foundation, The California Endowment, National Coalition for Health Integration, United Healthcare, and Sierra Health Foundation) played an instrumental role at each critical step in the expansion of our telehealth program. Federal funding from agencies including Agency for Healthcare Research and Quality, National 
Institutes of Health, and Health Resources and Services Administration have been vital for the success of many of our research investigators, including three faculty K08 awards (Mentored Clinical Scientist Development Award) focused exclusively on telehealth research.

In addition to funding tied to telehealth projects, in 2007 UC Davis received a commitment of $\$ 100$ million from the Gordon and Betty Irene Moore Foundation to start a new School of Nursing partially because of a demonstrated track record in utilizing technology to improve health outcomes and to implement innovative models of care. The Betty Irene Moore School of Nursing, approved in 2009, has created new collaborations and a broadened scholarly community with an expanded focus on chronic disease management and healthy aging.

In 2006, the voters of California passed a suite of infrastructure bonds, including Proposition 1D for education. This bond included language allocating \$200 million "to expand medical education in California with an emphasis on telemedicine." Each of the five Schools of Medicine in the University of California system received \$35 million for new facilities that achieve the goal of the bond; \$10 million was allocated to buy equipment for community sites in California. UC Davis' portion of the bond funding resulted in a new, 35,000 square foot, state-of-the-art facility that is dedicated to technology-enabled healthcare with high-speed telecommunications for clinical consultations, distance education and media production, and critical care and emergency services, as well as healthcare simulation training. It has telehealth consultation suites where faculty provides specialist outpatient and inpatient consultations to remote locations. The building has a floor that is a virtual hospital, with simulated operating rooms, inpatient ward, trauma room, and meeting space for debriefing. The entire building is designed to support medical education, health professional training, and lifelong learning, with an emphasis on the role of technology in the healthcare setting.

In 2007, UC Davis, on behalf of the State of California, received a 3-year, \$22.1 million award from the Federal Communications Commission to develop the California Telehealth Network (CTN) as part of the Federal Communications Commission's Rural Healthcare Pilot Program. The University of California system and the CTN team were also able to secure an additional $\$ 3.6$ million from foundations to support the implementation and match requirement of the network. The CTN is made up of a sophisticated high-performance network with multiprotocol label switching and has the ability to provide seamless connectivity to any network. The network currently has nearly 200 active remote sites with a goal of 860 sites by 2013 . The CTN further strengthens and supports the public service mission of the University by securing affordable broadband for healthcare facilities, an essential component of expanding access for people in California.

UC Davis, in collaboration with CTN, received an additional \$13.8 million project award as part of the Sustainable Broadband Adoption grant through the Broadband Technology Opportunities Program of the National Telecommunications and Information Administration.
This grant was designed to foster e-health broadband adoption by providing support for the initial implementation of CTN, develop an extensive training program to increase broadband application literacy for healthcare providers, and to establish model e-health communities to demonstrate successful technology-enabled healthcare. This grant enabled the establishment of 15 model e-health communities. The goal is to demonstrate best practices in the adoption of technology. UC Davis and educational partners also developed online training lessons in a wide variety of topics and created a consumer health education plan in collaboration with libraries and the UC Davis Extension Program and a workforce development program in partnership with community colleges.

UC Davis faculty and staff have also been instrumental in advancing policies necessary for telehealth development, including service on relevant committees and a liaison with the state legislature that ultimately led to the California Telemedicine Development Act of 1996. To date, we continue to work closely with policy organizations such as the Center for Connected Health Policy, the Health Resources and Services Administration-designated National Telehealth Policy Center, the Center for Telehealth and eHealth Law, and the American Telemedicine Association to inform future policies in telehealth. The philosophy of the CHT includes beliefs in a commitment to partnership between academia and policy development and in working closely with lawmakers and regulators on both the state and the federal level to promote a policy environment that supports the use of technology to improve access to care. Strategic research partnerships are also a fundamental component of our sustainability. UCDHS serves as the clinical lead for Center for Information Technology Research in the Interest of Society (CITRIS), one of the California State Institutes for Science and Innovation. CITRIS partners with more than 300 faculty and thousands of students from myriad departments at four University of California campuses (Berkeley, Davis, Merced, and Santa Cruz) and with industrial researchers from over 60 corporations. The mission is to create information technology solutions for several key societal issues: energy, healthcare delivery, the environment, and intelligent infrastructure. Telehealth is a visible component of the CITRIS agenda.

\section{Looking to the Future-Within UC Davis and for the Field of Telehealth}

In 2011 UCDHS embarked on an extensive process to create and implement a new Strategic Plan. Telehealth has been identified as an integral component to achieving several goals within the plan. For example, there is a specific tactic "to expand the strategic use of technologies to enhance research, education and clinical care by becoming national leader in informatics and health-care technology," another to "set the standard for applied informatics that catalyze technological innovation to improve health outcomes," and a third to "effectively deploy technologies to support the health systems goals and missions." To implement these actions, the CHT is in active discussions with the executive leadership of practice management board (faculty practice plan) to define the specific priorities that expand the reach of our faculty providers beyond the "brick and 


\section{NESBITT ET AL.}

mortar" clinics and to increase our faculty numbers without increasing capital costs.

The CHT is in the process of moving into its new state-of-the-art facility on the University's Sacramento campus. This facility is designed to enhance and complement UC Davis' long history and wide range of expertise in the field of telehealth. This new facility will create an optimal environment for our faculty to work in synergy with community partners to conduct research in newer telehealth technologies.

Also, as the Health System moves into a new era of healthcare financing, which include models such as accountable care organizations, we will be expanding our focus on home- and community-based technologies for chronic disease management to prevent hospital readmissions and improve quality of life and to use remote patient monitoring technologies to deliver timely care outside of the hospital and clinic setting. The Health System is currently conducting a large randomized study on the use of remote patient monitoring for congestive heart failure patients recently discharged from the hospital. Another clinical trial will investigate the effectiveness of remote patient monitoring on blood sugar control among patients with diabetes. More generally, healthcare reform is causing hospitals to merge and affiliate with larger medical centers. Additional local services and quality targets are likely to drive reimbursement, leading to more interest in hospital-based telemedicine on the part of affiliated partners. Current plans call for expanding telestroke, pediatric critical care program, electronic intensive care unit, and other hospital-based consultations to meet the expectations and requests from regional hospital partners. Also, our surgeons are beginning to develop models to provide telementoring (intraoperative telemedicine) during surgeries at other hospitals and are working on new telerobotic surgery platforms.

Looking toward the future, UC Davis is focused on technologyenabled health. The goal for the next decade will be to achieve synergy between the fields of telehealth, mobile health, electronic health informatics, informatics, and health information exchangewith an overarching vision to improve access and quality in a costeffective environment.

\section{Disclosure Statement}

No competing financial interests exist.

\section{REFERENCES}

1. Dharmar M, Marcin JP. A picture is worth a thousand words: Critical care consultations to emergency departments using telemedicine. Pediatr Crit Care Med 2009;10:606-607.

2. Marcin JP, Nesbitt TS, Kallas HJ, et al. Use of telemedicine to provide pediatric critical care inpatient consultations to underserved rural Northern California. J Pediatr 2004;144:375-380.

3. Nesbitt TS, Hilty DM, Kuenneth CA, Siefkin A. Development of a telemedicine program: A review of 1,000 videoconferencing consultations. West J Med 2000;173:169-174.

4. Marcin JP, Nesbitt TS, Cole SL, et al. Changes in diagnosis, treatment, and clinical improvement among patients receiving telemedicine consultations. Telemed J E Health 2005;11:36-43.

5. Marcin JP, Nesbitt TS, Struve S, Traugott C, Dimand RJ. Financial benefits of a pediatric intensive care unit based telemedicine program to a rural adult intensive care unit: Impact of keeping acutely ill and injured children in their local community. Telemed J E Health 2004;10(Suppl 2):S-1-S-5.

6. Nesbitt TS, Marcin JP, Daschbach MM, Cole SL. Perceptions of local healthcare quality in 7 rural communities with telemedicine. J Rural Health 2005;21: 79-85.
Address correspondence to: Madan Dharmar, MBBS, PhD Center for Health and Technology University of California Davis Health System Education Building 4610 X Street, Suite 2301 Sacramento, CA 95817

E-mail:mdharmar@ucdavis.edu

Received: November 20, 2012

Accepted: November 21, 2012 